\title{
A Large Group Decision Making Approach Based on TOPSIS Framework with Unknown Weights Information
}

Yupeng Li, Xiaozhen Lian*, Cheng Lu and Zhaotong Wang

Department of Industrial Engineering, School of Mines; Key Laboratory of Deep Coal Resource Mining, Ministry of Education of China; China University of Mining and Technology, Xuzhou, 221116, PR China

Corresponding Email: xzhen_1@163.com

\begin{abstract}
Large group decision making considering multiple attributes is imperative in many decision areas. The weights of the decision makers (DMs) is difficult to obtain for the large number of DMs. To cope with this issue, an integrated multiple-attributes large group decision making framework is proposed in this article. The fuzziness and hesitation of the linguistic decision variables are described by interval-valued intuitionistic fuzzy sets. The weights of the DMs are optimized by constructing a non-linear programming model, in which the original decision matrices are aggregated by using the interval-valued intuitionistic fuzzy weighted average operator. By solving the non-linear programming model with $\mathrm{MATLAB}^{\circledR}$, the weights of the DMs and the fuzzy comprehensive decision matrix are determined. Then the weights of the criteria are calculated based on the information entropy theory. At last, the TOPSIS framework is employed to establish the decision process. The divergence between interval-valued intuitionistic fuzzy numbers is calculated by interval-valued intuitionistic fuzzy cross entropy. A real-world case study is constructed to elaborate the feasibility and effectiveness of the proposed methodology.
\end{abstract}

Keywords. Multiple-attributes large group decision making, optimization of weights of DMs, non-linear programming model, TOPSIS.

\section{Introduction}

Operational management of many areas are consisted by series of decision making issues. Multiple-attributes group decision making (MAGDM) is widely applied to solve these operational management problems such as supplier selection [1], product concept evaluation [2], and facility location planning [3]. Somehow, in actual decision-making process, MAGDM cannot be accurately analyzed due to the complicated requirement of social and technological trend and the limitations of knowledge and experience of decision-makers (DMs), thus the participation of large group of DMs is 
imperative. The MAGDM with large group DMs is defined as multiple attributes large group decision making (MALGDM).

Research on MALGDM can be classified into two categories: clustering-based methods and consensus reaching process-based methods. The principle of the clustering-based method is to decrease the number of decision group by clustering the DMs into some communities, and DMs in the same community is weighted by the same weight. By extending the analytic hierarchy process (AHP) into the Euclidean vector space (VAHP), Zadir [4] defined the similarity measure between DMs, based on which, the DMs in MALGDM is partitioned into homogeneous groups by a clustering procedure. Bolloju [5] identified DMs into subgroups based on similarities in preferences and aggregated preferences within the same subgroups by using AHP. Based on the claimed that, many DMs in large group decision making had similar opinion, Tapia-Rosero et al. [6] proposed a shape-similarity-based clustering approach to improve the weighting process of DMs in MALGDM. To simplify the weighting process of DMs, Liu et al. [7] proposed a classification method for DMs based on the partial binary tree DEA-DA cyclic classification model. Then, to determine the weights of DMs in the cluster and the cluster weights, Liu et al. [8] proposed a two-layer model including Minimized Variance Model and an Entropy Weight Model. The essence of clustering-based methods is to decrease the sample of DMs, the main drawback of which is that, much useful weight information for individual DM will be eliminated. The consensus reaching process-based methods are regarded as the iterative procedure aimed at seeking a high degree of agreement among DMs' preferences before making a group decision. Palomares et al. [9] proposed a consensus iteration model complementing with a visual analysis tool of the overall consensus reaching process based on self-organizing maps, which facilitates the monitoring of the process performance across the time. Aiming at the lower consensus and the urgency of large group emergency decision making, $\mathrm{Xu}$ et al. [10] proposed and investigated a dynamical consensus method based on an exitdelegation mechanism. Zhang et al. [11] presented a new consensus-based group decision making model that an aggregation of individual priority-based aggregation mechanism to employ flexible methods for deriving each DM's priority and to avoid information loss caused by unifying heterogeneous information. Comparing with the clustering-based methods, the consensus reaching process-based methods can incorporate the opinions of all DMs. Inspired by the principle of consensus-based methods, a non-linear programming model optimizing the weights of the DMs through an iterative procedures among individual decision matrices and comprehensive decision matrix is proposed in this article.

Fuzziness always exists in MALGDM, which is described by interval-valued intuitionistic fuzzy set (IVIFS) in this article owning to its advantages to address the fuzziness and hesitation of DMs [12-13]. For the MALGDM problem, the determination of the weights of criteria and the selection of decision framework should also be seriously considered. As it can utilize the original decision information of DMs to reduce the subjectivity of some other weight methods such as AHP [14-15] and Delphi [16], the information entropy theory is employed to calculate the weights of the criteria. The TOPSIS framework is addressed to establish the decision process as it is an ideal points-based framework concerning both positive ideal solution (PIS) negative ideal solution (NIS) [17]. The interval-valued intuitionistic fuzzy weighting average (IIFWA) operator [18] is used to aggregate individual decision matrices into the comprehensive decision matrix. The interval-valued intuitionistic fuzzy (IVIF) cross-entropy [19] is used to measure the divergence between IVIFSs.

The reminder of this article is organized as follows. Section 2 provides some definitions. Section 3 proposes the MALGDM methodology. In section 4, an example is provided to illustrate the effectiveness of the proposed method. Section5 summarizes the major contributes of this article. 


\section{Preliminaries}

Definition 1[20] An IVIFS $A$ in a finite set of the universe of discourse $X=\left\{x_{1}, x_{2}, \ldots, x_{m}\right\}$ is: $A=\left\{\left\langle\left[u_{A}^{L}\left(x_{i}\right), u_{A}^{U}\left(x_{i}\right)\right],\left[v_{A}^{L}\left(x_{i}\right), v_{A}^{U}\left(x_{i}\right)\right]\right\rangle \mid x_{i} \in X\right\}$, and $\left\langle\left[u_{A}^{L}, u_{A}^{U}\right],\left[v_{A}^{L}, v_{A}^{U}\right]\right\rangle$ denotes the interval membership degree and non-membership degree of element $x_{i}$ belonging to $X$, respectively. And $\left[u_{A}^{L}, u_{A}^{U}\right] \in[0,1],\left[v_{A}^{L}, v_{A}^{U}\right] \in[0,1], u_{A}^{U}+v_{A}^{U} \leq 1$. The hesitation degree $\pi\left(x_{i}\right)$ of $x_{i}$ to $X$ is: $\pi\left(x_{i}\right)=\left[\pi_{A}^{L}, \pi_{A}^{U}\right]=\left[1-u_{A}^{U}-v_{A}^{U}, 1-u_{A}^{L}-v_{A}^{L}\right]$.

Definition 2[18] For an IVIFS $\tilde{a}_{k}=\left(\left[a_{k}, b_{k}\right],\left[c_{k}, d_{k}\right]\right)(k=1,2, \ldots, t)$, the IIFWA is defined as:

$$
\operatorname{IIFWA} A_{\lambda_{k}}\left(\tilde{a}_{1}, \tilde{a}_{2}, \ldots, \tilde{a}_{k}\right)=\left\{\left[1-\prod_{k=1}^{t}\left(1-a_{k}\right)^{\lambda_{k}}, 1-\prod_{k=1}^{t}\left(1-b_{k}\right)^{\lambda_{k}}\right],\left[\prod_{k=1}^{t} c_{k}^{\lambda_{k}}, \prod_{k=1}^{t} d_{k}^{\lambda_{k}}\right]\right\}
$$

where $\lambda=\left(\lambda_{1}, \lambda_{2}, \ldots, \lambda_{t}\right)$ is weight vector of DM and $\lambda_{k} \in[0,1], \sum_{k=1}^{t} \lambda_{k}=1$.

Definition 3[21] For two interval-valued intuitionistic fuzzy numbers (IVIFNs) $A=\left\{\left[u_{A}^{L}, u_{A}^{U}\right],\left[v_{A}^{L}, v_{A}^{U}\right]\right\}, B=\left\{\left[u_{B}^{L}\right.\right.$, $\left.\left.u_{B}^{U}\right],\left[v_{B}^{L}, v_{B}^{U}\right]\right\}$, the standard Euclidean distance between $A$ and $B$ is:

$$
d(A, B)=\frac{1}{m} \sum_{i=1}^{m}\left(\frac{1}{4}\left[\begin{array}{c}
\left|u_{A}^{L}-u_{B}^{L}\right|^{2}+\left|u_{A}^{U}-u_{B}^{U}\right|^{2}+\left|v_{A}^{L}-v_{B}^{L}\right|^{2}+\left|v_{A}^{U}-v_{B}^{U}\right|^{2}+ \\
\left|\pi_{A}^{L}-\pi_{B}^{L}\right|^{2}+\left|\pi_{A}^{U}-\pi_{B}^{U}\right|^{2}
\end{array}\right]\right)^{\frac{1}{2}}
$$

where $\pi_{\mathrm{A}}^{\mathrm{L}}=1-\mathrm{u}_{\mathrm{A}}^{\mathrm{U}}-\mathrm{v}_{\mathrm{A}}^{\mathrm{U}}, \pi_{\mathrm{A}}^{\mathrm{U}}=1-\mathrm{u}_{\mathrm{A}}^{\mathrm{L}}-\mathrm{v}_{\mathrm{A}}^{\mathrm{L}}, \pi_{\mathrm{B}}^{\mathrm{L}}=1-\mathrm{u}_{\mathrm{B}}^{\mathrm{U}}-\mathrm{v}_{\mathrm{B}}^{\mathrm{U}}, \pi_{\mathrm{B}}^{\mathrm{U}}=1-\mathrm{u}_{\mathrm{B}}^{\mathrm{L}}-\mathrm{v}_{\mathrm{B}}^{\mathrm{L}}$.

Definition 4[22] The entropy of $A=\left\langle\left[u_{A}^{L}, u_{A}^{U}\right],\left[v_{A}^{L}, v_{A}^{U}\right]\right\rangle$ is defined as:

$$
e(A)=1-d(A)
$$

where $d(A)=\left|\frac{u_{A}^{U}-v_{A}^{U}}{2}\right|+\left|\frac{L_{A}^{L}-v_{A}^{L}}{2}\right|$. The information entropy of the $j^{\text {th }}$ criterion $c_{j}$ is defined as:

$$
E_{j}=\frac{1}{n} \sum_{i=1}^{n} e_{i j}
$$

Then the weight of $c_{j}$ is calculated by:(1)

$$
\omega_{j}=\frac{1-E_{j}}{\sum_{j=1}^{m}\left(1-E_{j}\right)}=\frac{d_{j}}{\sum_{j=1}^{m} d_{j}}
$$

Definition 5[17] A symmetric form of IVIF cross-entropy between IVIFNs $A$ and $B$ is defined as:

$$
D^{*}(A, B)=D(A, B)+D(B, A)
$$

where:

$$
D(A, B)=\sum_{i=1}^{n}\left(\begin{array}{cc}
\frac{\underline{\mu}^{i}+\bar{\mu}_{A}^{i}+2-\underline{v}_{A}^{i}-\bar{v}_{A}^{i}}{4} \ln \frac{2\left(\underline{\mu}_{A}^{i}+\bar{\mu}_{A}^{i}+2--_{-}^{i}-\bar{v}_{A}^{i}\right)}{\underline{\mu}_{-A}^{i}+\bar{\mu}_{A}^{i}+2-\underline{v}_{A}^{i}-\bar{v}_{A}^{i}+\mu_{-}^{i}+\bar{\mu}_{B}^{i}+2-\underline{v}_{B}^{i}-\bar{v}_{B}^{i}}+ \\
\frac{\underline{v}_{A}^{i}+\bar{v}_{A}^{i}+2-\underline{\mu}_{A}^{i}-\bar{\mu}_{A}^{i}}{4} \ln \frac{2\left(\underline{v}_{A}^{i}+\bar{v}_{A}^{i}+2-\underline{\mu}_{-}^{i}-\bar{\mu}_{A}^{i}\right)}{\underline{v}_{A}^{i}+\bar{v}_{A}^{i}+2-\underline{\mu}_{-}^{i}-\bar{\mu}_{A}^{i}+\underline{v}_{B}^{i}+\bar{v}_{B}^{i}+2-\underline{\mu}_{-}^{i}-\bar{\mu}_{B}^{i}}
\end{array}\right)
$$

\section{Methodology}

The mathematical description of the MALGDM can be defined as following. The alternative set is: $X=\left\{x_{1}, x_{2}, \ldots\right.$, $\left.x_{i}, \ldots, x_{n}\right\},(i=1,2, \ldots, n)$, the evaluation criteria set is: $C=\left\{c_{1}, c_{2}, \ldots, c_{j}, \ldots, c_{m}\right\},(j=1,2, \ldots, m)$, the weights of $C$ is: $\Omega=\left\{\omega_{1}, \omega_{2}, \ldots, \omega_{j}, \ldots, \omega_{m}\right\},(j=1,2, \ldots, m)$, and the DM set is: $E=\left\{e_{1}, e_{2}, \ldots, e_{k}, \ldots, e_{t}\right\},(k=1,2, \ldots, t)$, the weight of $E$ is: $\Lambda=\left\{\lambda_{1}, \lambda_{2}, \ldots, \lambda_{k}, \ldots, \lambda_{t}\right\},(k=1,2, \ldots, t) . \widetilde{\boldsymbol{R}}_{\boldsymbol{k}}=\left(\tilde{r}_{i j k}\right)_{n \times m}$ is the interval-valued intuitionistic fuzzy matrix given by $e_{k}$, 
where $\tilde{r}_{i j k}=\left\langle\left[u_{k}^{L}\left(x_{i}\right), u_{k}^{U}\left(x_{i}\right)\right],\left[v_{k}^{L}\left(x_{i}\right), v_{k}^{U}\left(x_{i}\right)\right]\right\rangle$ is the ratings given by $e_{k}$ with regard to $c_{j}$ on $x_{i}$. Then the logic flow of the proposed methodology is demonstrated in Figure 1.

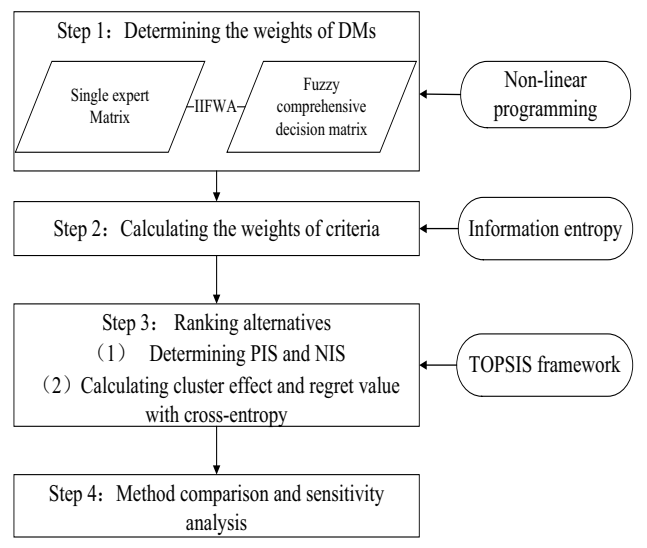

Fig 1. The logic flow of the proposed MGLGDM methodology.

The problem can be solved through three phases in detail.

Step 1: Calculating $\Lambda$ by using non-linear programming optimization model.

The optimization model of $\Lambda$ is constructed as following:

$$
\begin{aligned}
& \min \sum_{k=1}^{t} d\left(\widetilde{\boldsymbol{R}}_{\boldsymbol{k}}, \widetilde{\boldsymbol{R}}\right) \\
& \text { s.t. }\left\{\begin{array}{l}
0 \leq \lambda_{k} \leq 1 \\
\sum_{k=1}^{t} \lambda_{k}=1
\end{array}\right.
\end{aligned}
$$

where $d\left(\widetilde{\boldsymbol{R}}_{\boldsymbol{k}}, \widetilde{\boldsymbol{R}}\right)$ is the Euclidean distance (Equation (2)) between $\widetilde{\boldsymbol{R}}_{\boldsymbol{k}}$ and the fuzzy comprehensive decision matrix $\widetilde{\boldsymbol{R}}$ :

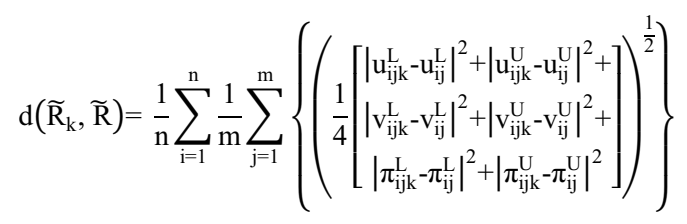

By Equation (9), the objective function is transformed to: $\min \sum_{k=1}^{t} d\left(\widetilde{\boldsymbol{R}}_{\boldsymbol{k}}, \widetilde{\boldsymbol{R}}\right)=$

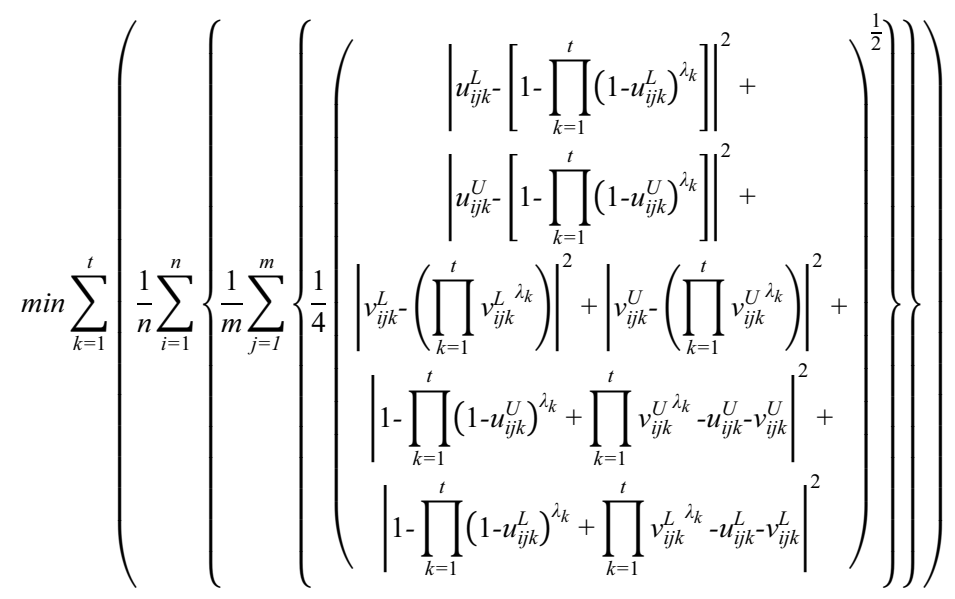




$$
\text { s.t. }\left\{\begin{array}{c}
0 \leq \lambda_{k} \leq 1 \\
\sum_{k=1}^{t} \lambda_{k}=1
\end{array}\right.
$$

Solve the model through MATLAB ${ }^{\circledR}$ to obtain the optimal solution of $\Lambda$. Then the fuzzy comprehensive decision matrix $\widetilde{\boldsymbol{R}}$ is acquired through IIFWA as:

$$
\boldsymbol{R}=\left(r_{i j}\right)_{n \times m}=\left[\begin{array}{ccc}
\left(\left[u_{11}^{L}, u_{11}^{U}\right],\left[v_{11}^{L}, v_{11}^{U}\right]\right) & \cdots & \left(\left[u_{1 m}^{L}, u_{1 m}^{U}\right],\left[v_{1 m}^{L}, v_{1 m}^{U}\right]\right) \\
\vdots & \ddots & \vdots \\
\left(\left[u_{n 1}^{L}, u_{n 1}^{U}\right],\left[v_{n 1}^{L}, v_{n 1}^{U}\right]\right) & \cdots & \left(\left[u_{n m}^{L}, u_{n m}^{U}\right],\left[v_{n m}^{L}, v_{n m}^{U}\right]\right)
\end{array}\right]_{n \times m}
$$

Step 2: Calculate the weight of criteria.

Calculating $\boldsymbol{\Omega}$ based on Definition 4 .

Step3: Alternative ranking.

(1) Determining the PIS and the NIS.

The comprehensive decision matrix $R_{i}$ of $x_{i}$ is denoted as:

$$
R_{i}=\left\{r_{i 1}, r_{i 2}, \ldots, r_{i m}\right\}=\left\{\begin{array}{c}
\left\langle\left[u_{i 1}^{L}, u_{i 1}^{U}\right],\left[v_{i 1}^{L}, v_{i 1}^{U}\right]\right\rangle,\left\langle\left[u_{i 2}^{L}, u_{i 2}^{U}\right],\left[v_{i 2}^{L}, v_{i 2}^{U}\right]\right\rangle, \\
\ldots,\left\langle\left[u_{i m}^{L}, u_{i m}^{U}\right],\left[v_{i m}^{L}, v_{i m}^{U}\right]\right\rangle
\end{array}\right\} .
$$

The PIS and the NIS are denoted in the form of IVIFS as, respectively:

$$
\begin{aligned}
P I S & =\left\{\begin{array}{c}
\left\langle\max _{i=1,2, \ldots, n}\left[u_{i 1}^{L}, u_{i 1}^{U}\right], \min _{i=1,2, \ldots, n}\left[v_{i 1}^{L}, v_{i 1}^{U}\right]\right\rangle,\left\langle_{i=1,2, \ldots, n}\left[u_{i 2}^{L}, u_{i 2}^{U}\right], \min _{i=1,2, \ldots, n}\left[v_{i 2}^{L}, v_{i 2}^{U}\right]\right\rangle, \\
\ldots,\left\langle_{i=1,2, \ldots, n}\left[u_{i m}^{L}, u_{i m}^{U}\right], \min _{i=1,2, \ldots, n}\left[v_{i m}^{L}, v_{i m}^{U}\right]\right\rangle
\end{array}\right\} \\
& =\left\{\left\langle\left[u_{i 1}^{L+}, u_{i 1}^{U+}\right],\left[v_{i 1}^{L+}, v_{i 1}^{U+}\right]\right\rangle,\left\langle\left[u_{i 2}^{L+}, u_{i 2}^{U+}\right],\left[v_{i 2}^{L+}, v_{i 2}^{U+}\right]\right\rangle, \ldots,\left\langle\left[u_{i m}^{L+}, u_{i m}^{U+}\right],\left[v_{i m}^{L+}, v_{i m}^{U+}\right]\right\rangle\right\} . \\
N I S & =\left\{\begin{array}{c}
\left.\left\langle\min _{i=1,2, \ldots, n}\left[u_{i 1}^{L}, u_{i 1}^{U}\right], \max _{i=1,2, \ldots, n}\left[v_{i 1}^{L}, v_{i 1}^{U}\right]\right\rangle, \bigcup_{i=1,2, \ldots, n}\left[u_{i 2}^{L}, u_{i 2}^{U}\right], \max _{i=1,2, \ldots, n}\left[v_{i 2}^{L}, v_{i 2}^{U}\right]\right\rangle, \\
\left.\ldots, \min _{i=1,2, \ldots, n}\left[u_{i m}^{L}, u_{i m}^{U}\right], \max _{i=1,2, \ldots, n}\left[v_{i m}^{L}, v_{i m}^{U}\right]\right\rangle
\end{array}\right\} \\
& =\left\{\left\langle\left[u_{i 1}^{L-}, u_{i 1}^{U-}\right],\left[v_{i 1}^{L-}, v_{i 1}^{U-}\right]\right\rangle,\left\langle\left[u_{i 2}^{L-}, u_{i 2}^{U-}\right],\left[v_{i 2}^{L-}, v_{i 2}^{U-}\right]\right\rangle, \ldots,\left\langle\left[u_{i m}^{L-}, u_{i m}^{U-}\right],\left[v_{i m}^{L-}, v_{i m}^{U-}\right]\right\rangle\right\} .
\end{aligned}
$$

(2) Calculate the weighted cross-entropy and relative closeness coefficient between each alternative and the PIS or NIS. $D^{*}\left(R_{i}, P I S\right)$ is the weighted cross-entropy between each alternative and the PIS. $D^{*}\left(R_{i}, N I S\right)$ is the weighted cross-entropy between each alternative and the NIS in the following. Then, the relative closeness degree (RCD) of each alternative is calculated, where $0 \leq \theta \leq 1$, and $\theta$ is the attention degree. Finally, the alternatives are ranked based on $R C D_{i}$ $(i=1,2, \ldots, n)$. The bigger the $R C D_{i}$ is, the better the alternative $x_{i}$ is.

$$
\begin{gathered}
R C D_{i}=\frac{\theta D^{*}\left(R_{i}, N I S\right)}{\theta D^{*}\left(R_{i}, P I S\right)+(1-\theta) D^{*}\left(R_{i}, P I S\right)} \\
D^{*}\left(R_{i}, P I S\right)=D\left(R_{i}, P I S\right)+D\left(P I S, R_{i}\right) \\
D\left(R_{i}, P I S\right)=\sum_{j=1}^{m}\left(\begin{array}{c}
w_{j} \frac{u_{i j}^{L}+u_{i j}^{U}+2-v_{i j}^{L}-v_{i j}^{U}}{4} \ln \frac{2\left(u_{i j}^{L}+u_{i j}^{U}+2-v_{i j}^{L}-v_{i j}^{U}\right)}{u_{i j}^{L}+u_{i j}^{U}-v_{i j}^{L}-v_{i j}^{U}+u_{i j}^{L+}+u_{i j}^{U+}-v_{i j}^{L+}-v_{i j}^{U+}+4}+ \\
w_{j} \frac{v_{i j}^{L}+v_{i j}^{U}+2-u_{i j}^{L}-u_{i j}^{U}}{4} \ln \frac{2\left(v_{i j}^{L}+v_{i j}^{U}+2-u_{i j}^{L}-u_{i j}^{U}\right)}{v_{i j}^{L}+v_{i j}^{U}-u_{i j}^{L}-u_{i j}^{U}+v_{i j}^{L+}+v_{i j}^{U+}-u_{i j}^{L+}-u_{i j}^{U+}+4}
\end{array}\right)
\end{gathered}
$$

\section{Case study and comparison analysis}

\subsection{Case study}


Due to the decline of traditional manufacturing industry, automobile manufacturing enterprises are confronted with high cost and low margin pressure. Some enterprises even are confronted with the danger of survival in the fierce competition environment. Under this circumstance, a growing number of enterprises are preparing customer-oriented 5S (Sale, Spare-part, Service, Survey, Sustainability) service. There are four alternatives providing 5S service, denoted as $x_{1}, x_{2}$, $x_{3}, x_{4}$ according to their own industry and management characteristics. The criteria of four alternatives $C=\left\{c_{1}, c_{2}, c_{3}, c_{4}\right\}$ are: the ability of satisfying customer demand, the controlling ability of operating costs, the sustainable development ability, and the enhancing ability of production and sales. The initial interval-valued intuitionistic fuzzy decision matrices of fifteen DMs are listed in Table 1.

According to the proposed MALGDM methodology, the alternatives are ranked through three steps in the following:

Step 1: The optimized model of the weights of DMs is constructed by using Equation (8) (10). The iteration number is set as 1000, the optimization target value is eventually obtained as 6.467 as shown in Figure 2. The weights of the DMs as shown in Table 2. The fuzzy comprehensive decision matrix $\widetilde{\boldsymbol{R}}$ is obtained using Equation (1), and the PIS and the NIS are determined using Equation (13) (14) as shown in Table 3.

Step 2: The weights of the criteria are obtained using Equation (4) (5), the results are listed in Table 4.

Step 3: The cross-entropy and relative closeness degree of alternatives are calculated by using Equation (15) (17), which are listed in Table 5.

According to the RCDs of each alternative, the alternatives are ranked as: $x_{3}>x_{2}>x_{1}>x_{4}$.

Table 1. The initial fuzzy decision matrices.

\begin{tabular}{|c|c|c|c|c|c|}
\hline & & $c_{1}$ & $c_{2}$ & $c_{3}$ & $c_{4}$ \\
\hline \multirow{16}{*}{$e_{1}$} & \multirow{4}{*}{$x_{1}$} & $<[0.5,0$. & $<[0.4,0$. & $<[0,3.0$. & $<[0.40$ \\
\hline & & $6]$ & 51 & 41 & \\
\hline & & {$\left[\begin{array}{llll}0 & 2 & 0 & 3\end{array}\right]$} & {$\left[\begin{array}{llll}0 & 2 & 0 & 3\end{array}\right]$} & {$\left[\begin{array}{lllll}0 & 3 & 0 & 4\end{array}\right]$} & {$\left[\begin{array}{llllll}0 & 3 & 0 & 4\end{array}\right]$} \\
\hline & & $>$ & $>$ & $>$ & $>$ \\
\hline & \multirow[t]{4}{*}{$x_{2}$} & $<[0.3,0$. & $<[0.5,0$. & $<[0.6,0$. & $<[0.6,0$. \\
\hline & & 4], & 6], & 7], & 7], \\
\hline & & {$[0.1,0.2]$} & {$[0.3,0.4]$} & {$[0.2,0.3]$} & {$[0.1,0.2]$} \\
\hline & & $>$ & $>$ & $>$ & $>$ \\
\hline & \multirow[t]{4}{*}{$x_{3}$} & $<[0.4,0$. & $<[0.3,0$. & $<[0.5,0$. & $<[0.7,0$. \\
\hline & & 5], & 4], & 6], & 8], \\
\hline & & {$[0.2,0.3]$} & {$[0.3,0.4]$} & {$[0.2,0.3]$} & {$[0.1,0.2]$} \\
\hline & & $>$ & $>$ & $>$ & $>$ \\
\hline & \multirow[t]{4}{*}{$x_{4}$} & $<[0.6,0$. & $<[0.5,0$. & $<[0.4,0$. & $<[0.3,0$. \\
\hline & & 7], & 6], & 5], & 4], \\
\hline & & {$[0.1,0.2]$} & {$[0.1,0.2]$} & {$[0.1,0.2]$} & {$[0.2,0.3]$} \\
\hline & & $>$ & $>$ & $>$ & $>$ \\
\hline & & & & & \\
\hline \multirow{3}{*}{$e_{1}$} & \multirow{3}{*}{$x_{1}$} & $<[0.5,0$. & $<[0.5,0$. & $<[0.2,0$. & $<[0.3,0$. \\
\hline & & 6], & 6], & 3], & $4]$ \\
\hline & & {$[0.2,0.3]$} & {$[0.2,0.3]$} & {$[0.4,0.5]$} & {$[0.4,0.5]$} \\
\hline
\end{tabular}




\begin{tabular}{|c|c|c|c|c|c|}
\hline & $>$ & $>$ & $>$ & $>$ \\
\hline \multirow{4}{*}{$x_{2}$} & $<[0.4,0$. & $<[0.8,0$. & $<[0.5,0$. & $<[0.7,0$. \\
& $5]$, & $9]$, & $6]$, & $8]$, \\
& {$[0.1,0.2]$} & {$[0,0.1]>$} & {$[0.3,0.4]$} & {$[0.1,0.2]$} \\
\hline \multirow{4}{*}{$x_{3}$} & $>$ & & $>$ & $>$ \\
& {$[0.6,0$.} & $<[0.6,0$. & $<[0.3,0$. & $<[0.4,0$. \\
& {$[0.2,0.3]$} & {$[0.2,0.3]$} & {$[0.5,0.6]$} & {$[0.2,0.3]$} \\
& $>$ & $>$ & $>$ & $>$ \\
\hline \multirow{4}{*}{$x_{4}$} & $<[0.4,0$. & $<[0.4,0$. & $<[0.4,0$. & $<[0.3,0$. \\
& $5]$, & $5]$, & $5]$, & $4]$, \\
& {$[0.4,0.5]$} & {$[0.3,0.4]$} & {$[0.3,0.4]$} & {$[0.3,0.4]$} \\
& $>$ & $>$ & $>$ & $>$ \\
\hline
\end{tabular}

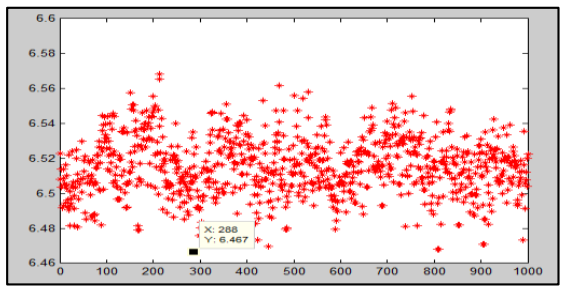

Fig. 2. The optimal value of the target function.

Table 2. The optimized results of weights of DMs.

\begin{tabular}{|c|c|c|c|c|c|}
\hline$e_{k}$ & $\lambda_{k}$ & $e_{k}$ & $\lambda_{\mathrm{k}}$ & $e_{k}$ & $\lambda_{k}$ \\
\hline$e_{1}$ & 0.124 & $e_{6}$ & 0.000 & $e_{11}$ & 0.012 \\
& 5 & & 7 & & 6 \\
\hline$e_{2}$ & 0.028 & $e_{7}$ & 0.108 & $e_{12}$ & 0.103 \\
\hline & 4 & 0 & & 5 \\
\hline$e_{3}$ & 0.070 & $e_{8}$ & 0.039 & $e_{13}$ & 0.071 \\
\hline$e_{4}$ & 0.113 & $e_{9}$ & 0.031 & $e_{14}$ & 0.003 \\
\hline$e_{5}$ & 0.139 & $e_{10}$ & 0.107 & $e_{15}$ & 0.047 \\
\hline
\end{tabular}

Table 3. The comprehensive decision matrix and the PIS(NIS).

\begin{tabular}{|c|c|c|c|c|}
\hline & $c_{1}$ & $c_{2}$ & $c_{3}$ & $c_{4}$ \\
\hline \multirow{4}{*}{$x_{1}$} & $<[0.543,0$. & $<[0.585,0$. & $<[0.538,0$. & $<[0.526,0$. \\
& $63]$, & $672]$, & $626]$, & $669]$, \\
& {$[0.236,0.3$} & {$[0.0,0.308$} & {$[0.0,0.335$} & {$[0.0,0.289$} \\
& $27]>$ & ]$>$ & ]$>$ & ]$>$ \\
\hline
\end{tabular}




\begin{tabular}{|c|c|c|c|c|}
\hline$x_{2}$ & $\begin{array}{c}<[0.585,0 . \\
696], \\
{[0.109,0.1} \\
86]>\end{array}$ & $\begin{array}{c}<[0.495,0 . \\
637], \\
{[0.0,0.344} \\
]>\end{array}$ & $\begin{array}{c}<[0.496,0 . \\
583], \\
{[0.0,0.276} \\
]>\end{array}$ & $\begin{array}{c}<[0.457,0 . \\
654], \\
{[0.166,0.2} \\
93]>\end{array}$ \\
\hline$x_{3}$ & $\begin{array}{c}<[0.501,0 . \\
578], \\
{[0.0,0.323} \\
]>\end{array}$ & $\begin{array}{c}<[0.544,0 . \\
677], \\
{[0.0,0.277} \\
\quad>\end{array}$ & $\begin{array}{c}<[0.513,0 . \\
604], \\
{[0.0,0.319} \\
]>\end{array}$ & $\begin{array}{c}<[0.540,0 . \\
673], \\
{[0.0,0.282} \\
]>\end{array}$ \\
\hline$x_{4}$ & $\begin{array}{c}<[0.586,0 . \\
667], \\
{[0.0,0.32]} \\
>\end{array}$ & $\begin{array}{c}<[0.562,0 . \\
667], \\
{[0.0,0.289} \\
]>\end{array}$ & $\begin{array}{c}<[0.472,0 . \\
564], \\
{[0.223,0.4} \\
13]>\end{array}$ & $\begin{array}{c}<[0.445,0 . \\
618], \\
{[0.0,0.348} \\
]>\end{array}$ \\
\hline PIS & $\begin{array}{c}<[0.586 \\
0.696] \\
{[0.0} \\
0.186]>\end{array}$ & $\begin{array}{c}<[0.585,0 . \\
677], \\
{[0.0,0.27} \\
6]>\end{array}$ & $\begin{array}{c}<[0.539,0 . \\
626] \\
{[0.0} \\
0.277]>\end{array}$ & $\begin{array}{c}<[0.540, \\
0.673], \\
{[0.0,0.28} \\
2]>\end{array}$ \\
\hline $\begin{array}{c}N I \\
S\end{array}$ & $\begin{array}{c}<[0.501,0 . \\
578], \\
{[0.236,0.3} \\
3]>\end{array}$ & $\begin{array}{c}<[0.495,0 . \\
637], \\
{[0 ., 0.344]} \\
>\end{array}$ & $\begin{array}{c}<[0.472,0 . \\
565], \\
{[0.223,0.4} \\
13]>\end{array}$ & $\begin{array}{c}<[0.445, \\
0.618] \\
{[0.166} \\
0.348]>\end{array}$ \\
\hline
\end{tabular}

Table 4. The weights of the criteria.

\begin{tabular}{|c|c|c|c|c|}
\hline & $c_{1}$ & $c_{2}$ & $c_{3}$ & $c_{4}$ \\
\hline$d_{j}$ & 1.6472 & 1.8100 & 1.4168 & 1.8100 \\
& & 5 & 5 & 5 \\
\hline$\omega_{j}$ & 0.255 & 0.279 & 0.218 & 0.248 \\
\hline
\end{tabular}

Table 5. The cross-entropy and RCD of alternatives.

\begin{tabular}{|c|c|c|c|c|}
\hline & \multicolumn{4}{|c|}{ Alternatives } \\
\hline & $x_{1}$ & $x_{2}$ & $x_{3}$ & $x_{4}$ \\
\hline$D^{*}\left(R_{i}, P I S\right)$ & 0.004 & 0.002 & 0.002 & 0.005 \\
& 7 & 7 & 2 & 2 \\
\hline$D^{*}\left(R_{i}, N I S\right)$ & 0.005 & 0.006 & 0.006 & 0.004 \\
\hline & 8 & 7 & 8 & 1 \\
\hline$R C D_{i}$ & 0.448 & 0.282 & 0.247 & 0.561 \\
\hline Ranking & 5 & 8 & 6 & 4 \\
order & 3 & 2 & 1 & 4 \\
\hline
\end{tabular}


The sensitivity of the proposed methodology is analyzed in this section by adjusting the original evaluation data [23]. For the highest importance degree, the hesitancy degree of $c_{2}$ in Table 4 is reduced to zero. The cross-entropy and the RCDs of each alternative are calculated by the proposed approach, the results are listed in Table 6 . For comparison, the weighted Euclidean distance and RCDs of each alternative are calculated by using Equation (9), the results are listed in Table 7. The Radar Map of the three kinds of ranking results is shown in Figure 3 and finally the ideal alternative is $x_{3}$. The ranking order does not change compared to the original order which can indicate that the applicability of the proposed approach is acceptable. Yet, the ranking order obtained by the weighted Euclidean distance under TOPSIS framework changes, that is, the ranks of $x_{1}$ and $x_{2}$ are swapped, which indicates that the proposed methodology is effective.

Table 6. RCD of alternatives calculating by the proposed method.

\begin{tabular}{|c|c|c|c|c|}
\hline & \multicolumn{4}{|c|}{ Alternative } \\
\hline & $x_{1}$ & $x_{2}$ & $x_{3}$ & $x_{4}$ \\
\hline$D^{*}\left(R_{i}, P I S\right)$ & 0.004 & 0.004 & 0.002 & 0.005 \\
7 & 1 & 7 & 3 \\
\hline$D^{*}\left(R_{i}, N I S\right)$ & 0.007 & 0.006 & 0.006 & 0.004 \\
\hline$R$ & 8 & 9 & 8 \\
\hline$R C D_{i}$ & 0.382 & 0.379 & 0.279 & 0.523 \\
\hline $\begin{array}{c}\text { Ranking } \\
\text { order }\end{array}$ & 3 & 2 & 1 & 4 \\
\hline
\end{tabular}

Table 7. RCD of alternatives calculating by Euclidean distance.

\begin{tabular}{|c|c|c|c|c|}
\hline & \multicolumn{4}{|c|}{ Alternative } \\
\hline & $x_{1}$ & $x_{2}$ & $x_{3}$ & $x_{4}$ \\
\hline$D\left(R_{i}, P I S\right)$ & 0.002 & 0.004 & 0.000 & 0.003 \\
& 5 & 4 & 53 & 4 \\
\hline$D\left(R_{i}, N I S\right)$ & 0.005 & 0.002 & 0.003 & 0.003 \\
& 4 & 0 & 3 & 2 \\
\hline$R C D_{i}$ & 0.319 & 0.687 & 0.138 & 0.517 \\
\hline Ranking & 9 & 2 & 4 & 7 \\
order & 2 & 4 & 1 & 3 \\
\hline
\end{tabular}

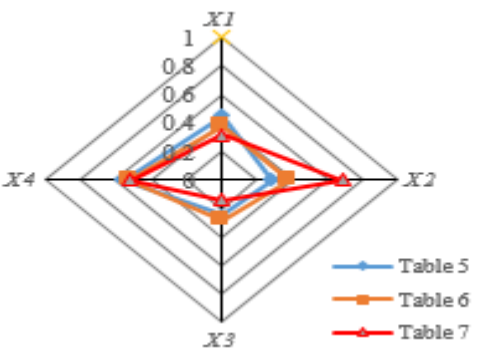

Fig 3. The three kinds of ranking result. 


\section{Conclusion}

This paper reviews approaches dealing with MALGDM problem which is difficult to obtain the weights of the DMs and proposes an integrated MALGDM framework. The main contributions are as follows.

(1) First, the weights of the DMs are optimized by constructing a non-linear programming model, in which the original decision matrices are aggregated by using IIFWA operator. By solving the non-linear programming model with MATLAB $^{\circledR}$, the weights of the DMs and the comprehensive decision matrix are determined.

(2) Second, the IVIFS is introduced to describe the membership degree and non-membership degree of the DMs. The IVIF-cross entropy is employed to measure the divergence degree between IVIFNs and the information entropy is employed to determine weights of criteria under TOPSIS framework.

A selection problem of alternatives providing $5 \mathrm{~S}$ service is implemented as the case study. There are still some issues need to be considered in the future. For example, the consensus analysis can be introduced to increase the consensus between $\widetilde{\boldsymbol{R}}_{\boldsymbol{k}}$ and $\widetilde{\boldsymbol{R}}$.

\section{Acknowledgments}

The authors express sincere appreciation to the anonymous referees for their helpful comments to improve the quality of the paper.

This research is supported by the Fundamental Research Funds for the Central Universities (grant No. 2015QNA41), the National Natural Science Foundation, China (No. 51505480, 51475290), Natural Science Foundation of Jiangsu Province (No. BK20150197).

\section{References}

1. J. Qin, X. Liu, W. Pedrycz. A multiple attribute interval type-2 fuzzy group decision making and its application to supplier selection with extended LINMAP method . Soft Computing. 1-20 (2016).

2. D. Guha, D. Chakraborty. Fuzzy multi-attribute group decision making method to achieve consensus under the consideration of degrees of confidence of experts' opinions 3 . Computers \& Industrial Engineering. 4, 493-504 (2011).

3. J. Xiong, Y.W. Chen, K.W. Yang. A decision support model for multi-attribute group decision making using a multi-objective optimization approach. International Journal of Computational Intelligence Systems. 2, 337-353 (2013).

4. S. Zahir. Clusters in a group: Decision making in the vector space formulation of the analytic hierarchy process. European Journal of Operational Research. 3, 620-634 (1999).

5. N. Bolloju. Aggregation of analytic hierarchy process models based on similarities in decision makers' preferences ㄱ. European Journal of Operational Research. 3, 499-508 (2001).

6. A. Tapia-Rosero, Bronselaer A, Tré G D. A method based on shape-similarity for detecting similar opinions in group decision-making. Information Sciences. 258, 291-311 (2014).

7. B. Liu, Y Shen, X Chen. A partial binary tree DEA-DA cyclic classification model for decision makers in complex multi-attribute large-group interval-valued intuitionistic fuzzy decision-making problems. Information Fusion. 1, $119-130$ (2014).

8. B. Liu, Y. Shen, Y. Chen. A two-layer weight determination method for complex multi-attribute large-group decision-making experts in a linguistic environment. Information Fusion. 23, 156-165 (2015). 
9. I. Palomares, L. Martinez, F. Herrera. A Consensus Model to Detect and Manage Noncooperative Behaviors in Large-Scale Group Decision Making. IEEE Transactions on Fuzzy Systems. 3, 516-530 (2014).

10. X.H. Xu, X.Y. Zhong, X.H. Chen. A dynamical consensus method based on exit-delegation mechanism for large group emergency decision making. Knowledge-Based Systems. 86, 237-249 (2015).

11. F. Zhang, J. Ignatius, Y. Zhao. An improved consensus-based group decision making model with heterogeneous information. Applied Soft Computing. 35, 850-863 (2015).

12. K. Atanassov, G. Gargov. Interval valued intuitionistic fuzzy sets. Fuzzy Sets \& Systems. 3, 343-349 (1989).

13. S.P. Wan, G.L. Xu, F. Wang. A new method for Atanassov's interval-valued intuitionistic fuzzy MAGDM with incomplete attribute weight information. Information Sciences. 316, 329-347 (2015).

14. R. Al-Aomar. A combined AHP-entropy method for deriving subjective and objective criteria weights. International Journal of Industrial Engineering Theory Applications \& Practice. 1, 12-24 (2010).

15. Y. Yu, Y. Wu, N. Yu. Fuzzy comprehensive approach based on AHP and entropy combination weight for pipeline leak detection system performance evaluation// Systems Conference (SysCon), 2012 IEEE International. 1-6 (2012).

16. I.A. Curtis. Valuing ecosystem goods and services: a new approach using a surrogate market and the combination of a multiple criteria analysis and a Delphi panel to assign weights to the attributes. Ecological Economics. 3-4, 163-194 (2004).

17. Y. Li, X. Chu, D. Chu. An integrated approach to evaluate module partition schemes of complex products and systems based on interval-valued intuitionistic fuzzy sets. International Journal of Computer Integrated Manufacturing 7, 675-689 (2013).

18. K. Xu, J. Zhou, R. Gu. Approach for aggregating interval-valued intuitionistic fuzzy information and its application to reservoir operation. Expert Systems with Applications. 7, 9032-9035 (2011).

19. H. Zhang, L. Yu. MADM method based on cross-entropy and extended TOPSIS with interval-valued intuitionistic fuzzy sets. Knowledge-Based Systems. 2, 115-120 (2012).

20. K.T. ATANASSOV. Interval Valued Intuitionistic Fuzzy Sets. Intuitionistic Fuzzy Sets. Physica-Verlag HD. 343-349 1999 .

21. L.C. Jang, W.J. Kim, T. Kim. A Note on Distances between Interval-Valued Intuitionistic Fuzzy Sets. International Journal of Fuzzy Logic \& Intelligent Systems. 1, 8-11 (2011).

22. S. Wang. A Novel Multi-attribute Allocation Method Based on Entropy Principle. Journal of Software Engineering. 1, 16-20 (2012).

23. R. Simanaviciene, L. Ustinovichius. Sensitivity Analysis for Multiple Criteria Decision Making Methods: TOPSIS and SAW. Procedia - Social and Behavioral Sciences. 6, 7743-7744 (2010). 